\title{
TRAVERSAL TIMES OF MARKOV PROCESSES
}

\author{
BY R. V. CHACON AND BENTON JAMISON
}

There are two main results we wish to state which are proved in a series of papers starting with [1]. The first is that whenever a particle which is undergoing motion governed by a strong Markov process with stationary transition probabilities and without holding points traces out a fixed trajectory segment, it must trace it out in a fixed length of time depending only on the transition probabilities and on the trajectory segment. This theorem has as an immediate corollary the fact that a continuous Markov process on the real line which always moves in the same direction must do so deterministically. The second main result, an application of the first, is that if two processes have the same hitting probabilities and the second is a strong Markov process with stationary transition probabilities, then the first can be transformed into the second by a nonanticipating change of the time scale, without having to enlarge the $\sigma$-fields. This is a sharpening of the result given in [2] for two arbitrary processes with the same hitting probabilities and no holding points, but only in the case that the second process is stong Markov.

The first result may be stated formally as follows. There is a set $\Omega_{1} \subset \Omega$, with $P_{\mu}\left(\Omega_{1}\right)=1$ for all initial distributions $\mu$, such that if $H=\left\{X_{t}(\omega)\right.$ : $\omega \in$ $\Omega_{1}$ \} then $H$ is closed under shifts, and $H$ is consistently traced. That $H$ is closed under shifts means that if $X_{t}(\omega) \in H$, then $X_{t+s}(\omega) \in H$ for all $s \geqslant 0$. That $H$ is consistenly traced means that if $X_{t}(\omega)$ and $X_{t}\left(\omega^{\prime}\right)$ are in $H$ and trace out the same trajectory segment, there they must do so at the same rate: if $X_{t}(\omega), a \leqslant$ $t \leqslant b$ and $X_{t}\left(\omega^{\prime}\right), c \leqslant t \leqslant d$ determine the same trajectory segment, then $b-$ $a=d-c$ and $X_{a+s}(\omega)=X_{c+s}\left(\omega^{\prime}\right), 0 \leqslant s \leqslant b-a=d-c$.

As is customary in probability theory, path denotes a function of time having values in the state space and trajectory denotes the ordered set of points traversed by the path, so that, for example, if $\omega$ is fixed, $X_{t}(\omega)$ and $X_{t^{2}}(\omega)$ are different paths traversing the same trajectory. To carry out the proof of the first result, let $\tau_{1} \leqslant \tau_{2}$ be two stopping times with $\tau_{2}$ defined by the post- $\tau_{1}$ behavior of the process. First, the paths of the Markov process are grouped into equivalence classes consisting of those paths which traverse the same trajectory segment as $t$ ranges over the interval $\left[\tau_{1}, \tau_{2}\right)$, and then the probability measure of the process is disintegrated with respect to these equivalence classes. Next, it is shown that the time of traversal of the segment is, for almost all

Received by the editors December 13, 1978.

AMS (MOS) subject classifications (1970). Primary $60 \mathrm{~J} 25$.

() 1979 American Mathematical Society 
trajectory segments with $\tau_{1} \leqslant \tau_{2}$ fixed, a constant depending on the trajectory segment and the transition probabilities of the process. This dependence is such that the traversal time of trajectory segments is consistent in the sense that if a segment is the union of other segments, then its traversal time is the sum of the traversal times of its components. That the traversal time of a segment is constant is proved by first showing that it can be written as the sum of an arbitrarily large number of random variables, which, relative to the conditional measure on the trajectory segment, are not only independent but subject to the normality convergence criterion for triangular arrays, in the case that $\tau_{2}<+\infty$, and is therefore normal. Since the traversal time must be nonnegative, its normal distribution must degenerate at a constant. The idea of the proof that the traversal time is the sum of independent random variables is contained in the following observations. Let $\sigma_{1}$ be the first exit time from a ball of radius 1 centered at the location of the process at time 0 , and let $\sigma_{2}$ be the first exit time after $\sigma_{1}$ from a ball of radius 1 centered at the location of the process at time $\sigma_{1}$. The paths are grouped into equivalence classes indexed by the three coordinates $\xi=\left(X_{1}, X_{2}, X_{3}\right)$, where $X_{1}$ is the location of the path at time $0, X_{2}$ is the location of the path at time $\sigma_{1}$ and $X_{3}$ the location of the path at time $\sigma_{2}$ and then the measure $P$ on path space is disintegrated with respect to the equivalence classes. The Markov property as usually stated would give the conditional independence of $\sigma_{1}$ and $\sigma_{2}-\sigma_{1}$ given $X_{2}$, but what is needed to give independence of $\sigma_{1}$ and $\sigma_{2}-\sigma_{1}$ with respect to $P_{\xi}$ for almost all $\xi$ is the conditional independence of $\sigma_{1}$ and $\sigma_{2}-\sigma_{1}$ given $\xi=\left(X_{1}, X_{2}\right.$, $X_{3}$ ). This follows from the first part and the lemma that if $A$ and $B$ are conditionally independent given $C$ and if $A_{0} \subset A, B_{0} \subset B, C_{0}=C$ then $A$ is conditionally independent of $B \vee C_{0}$ given $A_{0} \vee B_{0} \vee C$.

If the condition that there are no holding points is removed, then it is shown that both results still hold when properly modified. The modification takes into account that for each holding point of the Markov process there is a parameter, depending only on the point, such that when the process reaches the point, it holds for an exponential holding time with that parameter. The process thus has more than one sample path in each trajectory segment having holding points. The process, when restricted to a trajectory segment, moves deterministically when it moves and holds exponentially at holding points with the proper parameter. The result so modified yield the Blumenthal, Getoor and McKean theorem directly.

BIBLIOGRAPHY

1. R. V. Chacon and Benton Jamison, $A$ fundamental property of Markov processes with an application to equivalence under time changes, Israel $\mathrm{J}$. Math.

2. - Processes with state-dependent hitting probabilities and their independence under time changes, Advances in Math.

DEPARTMENT OF MATHEMATICS, UNIVERSITY OF BRITISH COLUMBIA, VANCOUVER, BRITISH COLUMBIA, CANADA

DEPARTMENT OF MATHEMATICS, STATE UNIVERSITY OF NEW YORK AT ALBANY, ALBANY, NEW YORK 12222 